\title{
Identificación de peligros químicos en cuencas de abastecimiento de agua como instrumento para la evaluación del riesgo
}

\author{
Karen Alejandra Bueno-Zabala* \\ Andrea Pérez-Vida ${ }^{* *}$ \\ Patricia Torres-Lozada***
}

Recibido: 06/02/2013 - Aceptado: 06/09/2013

\begin{abstract}
Resumen
Las cuencas de abastecimiento son la primera y principal barrera de los sistemas de abastecimiento de agua potable (SAAP) y, por lo tanto, la identificación de peligros es un instrumento clave para apoyar la evaluación y gestión de los riesgos que puedan comprometer la calidad del agua potable. Este estudio identificó los peligros químicos presentes en la cuenca alta del río Cauca (Colombia), apoyándose en la construcción de matrices de priorización y en la caracterización de agua y sedimentos. De acuerdo con los usos del suelo se identificaron cinco sustancias prioritarias a controlar y 14 plaguicidas. La caracterización de los peligros químicos en agua y sedimentos mostró la presencia de algunos metales pesados y sustancias de interés sanitario que podrían representar riesgos a la salud, si los SAAP que se abastecen de esta fuente en el tramo evaluado, no cuentan con barreras de tratamiento adecuadas.
\end{abstract}

Palabras clave: calidad del agua, evaluación del riesgo, gestión de cuencas, identificación de peligros, sedimentos, sistema de abastecimiento de agua potable.

\footnotetext{
* Ing. Sanitaria, MSc. en Ing. énfasis Ing. Sanitaria y Ambiental. Joven Investigadora Colciencias, Grupo Investigación Estudio y Control de la Contaminación Ambiental, Universidad del Valle-Colombia. Calle 13 \# 100 - 00 Edificio 336. Teléfono: 3312175. karen.bueno@correounivalle.edu.co

** Ing. Sanitaria, MSc. y PhD. en Ing. énfasis Ing. Sanitaria y Ambiental, Universidad del Valle. Profesora Titular Universidad de Boyacá, Grupo Investigación Gestión del Recurso Hídrico. Tunja, Colombia. Cra. 2Este \#64-169. Teléfono: 7450000 Ext 6304. aperezv@uniboyaca.edu.co

*** Ing. Sanitaria Universidad del Valle, MSc. y PhD. en Ing. Civil, énfasis Hidráulica y Saneamiento Universidad de São Paulo-Brasil. Profesora Titular Universidad del Valle, Grupo Investigación Estudio y Control de la Contaminación Ambiental. Cali, Colombia. Calle 13 \# 100 - 00 Edificio 336. Teléfono: 3312175. patricia.torres@, correounivalle.edu.co
} 


\section{Identification of chemical hazards in supply watersheds as an instrument for risk evaluation}

\section{Abstract}

Supply watersheds are the first and foremost barrier of the potable water supply systems (PWSS) and, therefore, the identification of hazards is a key instrument for supporting the evaluation and management of risks that could compromise the quality of potable water. The chemical hazards of the upper watershed of the Cauca river (Colombia) were identified in this study, taking as a basis the construction of prioritization matrices and the characterization of water and sediments. According to the uses of the soil, five priority substances and fourteen pesticides that need to be controlled were identified. The characterization of the chemical hazards in water and sediments exhibited the presence of several heavy metals and substances of sanitary interest that could represent health risks if the PWSS supplied by this source in the evaluated section do not have adequate treatment barriers.

Key words: water quality, risk assessment, watershed management, hazard identification, sediments, potable water supply system. 


\section{INTRODUCCIÓN}

Los planes de seguridad del agua (PSA) permiten el aseguramiento de la calidad del agua en sistemas de abastecimiento de agua potable (SAAP) desde la fuente hasta el usuario final y se apoyan en la evaluación y gestión del riesgo [1] que pueden ser realizadas de forma cualitativa o cuantitativa [2], siendo la primera etapa la identificación de peligros. Un peligro es cualquier agente físico, biológico, químico o radiológico que puede afectar la salud pública, y un evento peligroso es aquella situación o actividad que introduce peligros o impide su eliminación en el SAAP; el riesgo es la probabilidad de ocurrencia de un evento peligroso en un tiempo determinado [1].

Para la identificación de peligros, se usan metodologías como la lluvia de ideas, experiencias del pasado, el análisis de la pregunta “¿qué pasa si...?", listas de verificación, entre otras. La elaboración de matrices de peligros y eventos peligrosos son herramientas que pueden apoyar la identificación de peligros [1,2], y se requiere, para las cuencas de abastecimiento, la recopilación de información de calidad de agua y la identificación de los impactos ocasionados por contaminación puntual (Ej.: descarga de aguas residuales) o difusa (Ej: escorrentía agrícola). Las fuentes de contaminación analizadas de forma individual pueden resultar insignificantes, pero en conjunto representan impactos adversos significativos, incrementando las concentraciones de sustancias con riesgo a la salud como los metales pesados y plaguicidas que tienden a ser adsorbidos en la superficie del material particulado, depositándose en los sedimentos [3,4] y que pueden transferirse hacia la columna de agua por factores como resuspensión durante picos de caudal en los ríos, disminución del $\mathrm{pH}$ generado por vertimientos mineros e industriales, cambios en condiciones redox y variaciones climáticas $[5,6]$.

La cuenca del río Cauca es un importante recurso hídrico del país, que abastece cerca del $25 \%$ de la población asentada en su área de influencia y el $80 \%$ de la población caleña; sin embargo, está impactada por usos del suelo causantes de peligros de tipo fisicoquímico asociados a la minería (oro, carbón, material de arrastre), vertimiento de aguas residuales industriales sin previo tratamiento y la escorrentía agrícola, que podrían incrementar el riesgo sanitario de la población en caso de no contarse con barreras de tratamiento que garanticen agua potable y segura. Teniendo en cuenta su importancia como fuente de abastecimiento para el municipio de Santiago de Cali y su creciente deterioro, en este estudio se identificaron los peligros químicos asociados a usos de suelo mediante la construcción de una matriz de priorización y la caracterización de agua y sedimentos como instrumentos para la evaluación del riesgo.

\section{MATERIALES Y MÉTODOS}

La figura 1 muestra el tramo de 135,7 km del río Cauca evaluado (jurisdicción de dos departamentos: Cauca y Valle del Cauca). La selección de los puntos de muestreo se 
fundamentó en el progresivo deterioro de la calidad del agua del río Cauca a partir del embalse Salvajina.

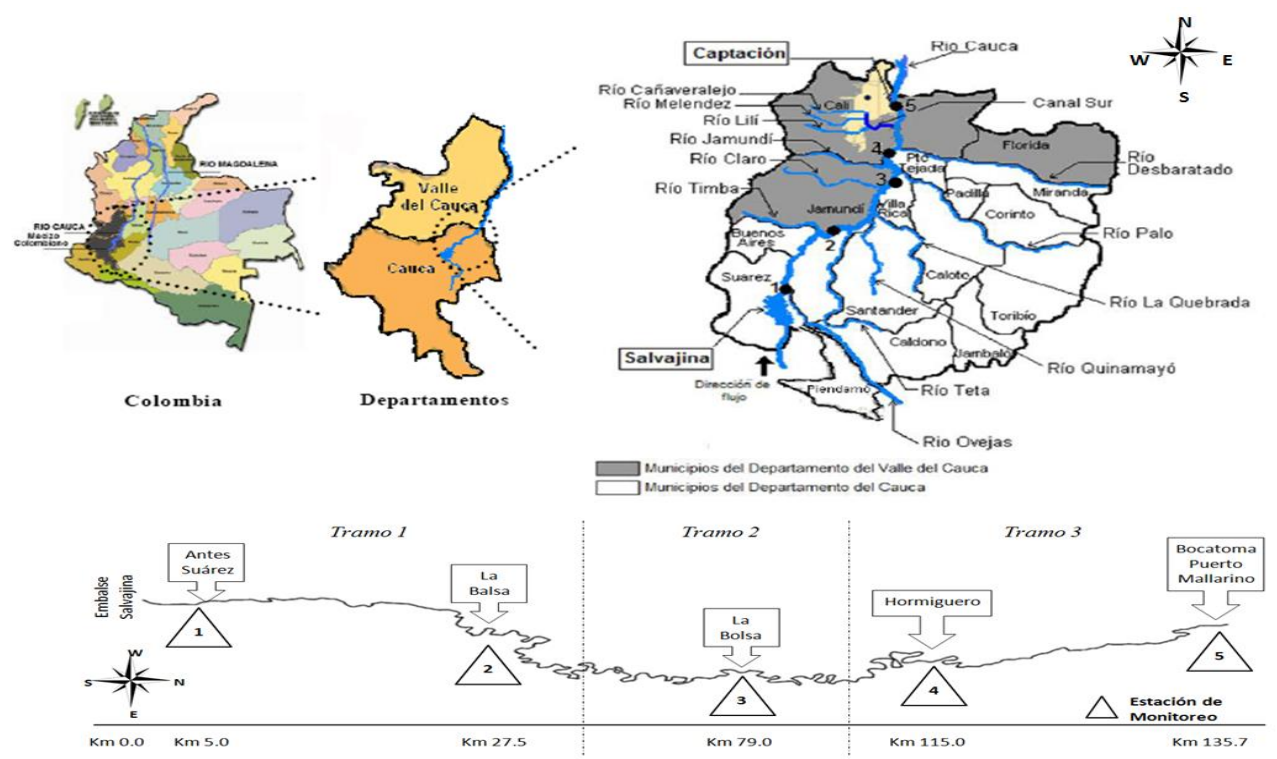

Figura 1. Localización de la zona de estudio.

Fuente: elaboración propia

La identificación de peligros químicos aportados por contaminación puntual (sector industrial) y difusa (minería y escorrentía agrícola) comprendió dos etapas: i) construcción de la matriz de priorización de peligros químicos, y ii) muestreo y caracterización de la calidad de agua y sedimentos.

\subsection{Construcción de matriz de priorización de peligros químicos}

Se recopiló información sobre las actividades que aportan peligros químicos a la cuenca como agricultura, minería y sector industrial. La información se obtuvo en fuentes como la Corporación Regional del Cauca (CRC), la empresa prestadora del servicio de agua potable EMCALI EICE ESP y DNP/MAVDT/ MHCP [7]. Adicionalmente, se recopilaron datos históricos de calidad fisicoquímica del agua del río Cauca y sus principales tributarios, suministrados por la Corporación Regional del Valle del Cauca (CVC) y estudios como los reportados por Escobar et al. [8] y Torres et al. [9].

Con relación a la agricultura, el uso de plaguicidas fue prioritario, y se destacaron 130 ingredientes activos ( 60 herbicidas, 38 insecticidas y 32 fungicidas) usados en los principales cultivos de la zona [10] de los cuales se priorizaron 6 herbicidas, 6 insec- 
ticidas y 2 fungicidas usando criterios como número de cultivos en que se emplea y características químicas (toxicidad, movilidad y persistencia del ingrediente activo). La tabla 1 resume la puntuación y clasificación de los criterios empleados en la matriz de priorización de los plaguicidas usados, y la tabla 2, el listado de plaguicidas seleccionados a partir de esta matriz.

Tabla 1. Criterios establecidos para priorizar los ingredientes activos de plaguicidas.

\begin{tabular}{|c|c|c|c|l|}
\hline \multirow{2}{*}{ Puntuación } & \multicolumn{4}{|c|}{ Criterio de priorización } \\
\cline { 2 - 5 } & $\begin{array}{c}\text { Categoría } \\
\text { Toxicológica }\end{array}$ & Persistencia & Movilidad & \multicolumn{1}{|c|}{ Número y tipo de cultivos } \\
\hline 5 & IA y IB & $>60$ días & Muy alta & $\begin{array}{l}\text { Uso en caña de azúcar y al menos } 3 \text { cultivos } \\
\text { adicionales o más de } 6 \text { cultivos }\end{array}$ \\
\hline 4 & II & $60-30$ días & Alta & $\begin{array}{l}\text { Uso en caña de azúcar y al menos } 2 \text { cultivos } \\
\text { adicionales o más de } 5 \text { cultivos }\end{array}$ \\
\hline 3 & II - III & $30-20$ días & Media & Uso en 4 cultivos \\
\hline 2 & III & $20-5$ días & Baja & Uso en 3 cultivos \\
\hline 1 & IV & $<5$ días & Nula & Uso en 2 cultivos \\
\hline
\end{tabular}

IA: producto extremadamente peligroso. IB: producto altamente peligroso. II: producto moderadamente peligroso. III: producto poco ligeramente peligroso. IV: producto que normalmente no representa peligro. ${ }^{\star}$ Grado de peligro ( 5 valor más alto; 1 valor más bajo) ${ }^{* *}$ Adaptado de Corra [11]

Fuente: elaboración propia

Tabla 2. Plaguicidas seleccionados a partir de la matriz de priorización.

\begin{tabular}{|c|c|c|c|c|c|c|}
\hline \multirow{3}{*}{$\begin{array}{c}\text { Cultivos } \\
\text { predominantes }\end{array}$} & \multicolumn{6}{|c|}{ Principales Plaguicidas } \\
\hline & \multicolumn{2}{|l|}{ Herbicidas } & \multicolumn{2}{|l|}{ Insecticidas } & \multicolumn{2}{|l|}{ Fungicidas } \\
\hline & Ingrediente activo & $P$ & Ingrediente activo & $P$ & Ingrediente activo & $P$ \\
\hline \multirow{5}{*}{$\begin{array}{l}\text { Caña, café, } \\
\text { plátano, arroz, } \\
\text { sorgo, soya, } \\
\text { maíz, yuca, } \\
\text { cacao, piña e } \\
\text { ilícitos }\end{array}$} & $\begin{array}{l}\text { Atrazina, Paraquat, } \\
\text { Picloram }\end{array}$ & 17 & Dimetoato, Metomil & 19 & Mancozeb & 18 \\
\hline & $\begin{array}{l}\text { Ametrina, Diuron, } \\
\text { Glifosato }\end{array}$ & 16 & Clorpirifos & 17 & Carbendazim & 16 \\
\hline & 2,4-D, Pendimethalina & 15 & $\begin{array}{l}\text { Carbofuran, fosfamidón, } \\
\text { metamidofos }\end{array}$ & 16 & Triadimefon & 15 \\
\hline & & & Carbaryl & 15 & Clorotalonil & 12 \\
\hline & & & Endosulfan* & 14 & & \\
\hline
\end{tabular}

P: Puntaje obtenido a partir de la suma de los cuatro criterios de priorización (toxicidad, movilidad, persistencia y número y tipo de cultivos). El valor más alto indica que es prioritaria su medición.

Fuente: elaboración propia

Respecto a minería y sector industrial, se tuvo en cuenta el número de unidades productivas -UP- (número de industrias y minas georreferenciadas) que descargan sus aguas residuales directamente al río Cauca o a través de los vertimientos de aguas 
residuales domésticas, y los productos químicos usados en los procesos productivos. A partir del procesamiento sistemático y análisis de la información, se elaboró un listado preliminar de los sectores productivos, y se encontraron 670 UP que utilizan 50 compuestos químicos. Debido a su aporte químico y los posibles riesgos a la salud, se priorizaron 604 UP que utilizan 20 compuestos químicos, que permitieron la elaboración de la matriz de priorización de peligros químicos, y su clasificación en 13 categorías asociadas a diferentes sectores productivos.

Las categorías fueron ordenadas de acuerdo con el número de UP; la primera es la que representa el mayor número y la última el menor; los 20 compuestos químicos fueron ordenados teniendo en cuenta el número total de UP que los utiliza en cada sector y que podrían ser aportados a la cuenca del río Cauca durante la descarga de los vertimientos líquidos a los afluentes tributarios o directamente al río Cauca. Los resultados de las matrices de priorización permitieron definir las variables químicas a ser consideradas durante las caracterizaciones de calidad de agua y sedimentos.

\subsection{Muestreo y caracterización de agua y sedimentos}

Se realizaron dos muestreos de calidad del agua (temporada lluviosa y de pocas lluvias), y se monitorearon los peligros químicos identificados en la matriz de priorización. Dado que los mapas de uso agrícola y georreferenciación de industrias elaborados (Figura 2) evidencian agricultura intensiva y como cultivo predominante la caña de azúcar y que la concentración del sector industrial y el mayor deterioro de la calidad del agua ocurren a partir de la estación 3 (La Bolsa), los sedimentos se caracterizaron solo en las estaciones Hormiguero y Bocatoma, ya que estas características indican una mayor probabilidad de encontrar sedimentos impactados por sustancias químicas a partir de la estación de Hormiguero. Basados en los resultados de la matriz de priorización de peligros químicos, en los sedimentos, se caracterizaron los metales pesados que se presentaron en más de 100 UP y que excedieran los valores de referencia para el uso de la fuente para consumo humano, previo tratamiento.

La toma y preservación de muestras se realizó de acuerdo con APHA et al. [12]; las muestras de agua se recolectaron en el punto medio del cauce a $30 \mathrm{~cm}$ de profundidad y los sedimentos se extrajeron de las márgenes del río para evitar las altas velocidades del centro $(\sim 1 \mathrm{~m} / \mathrm{s})$ que impiden la sedimentación [13] y en lugares permanentemente cubiertos de agua, sin turbulencias ni meandros. La muestra de sedimentos se compuso con submuestras (4) de 300 gramos, recolectadas con una draga Van Veen recomendada para fuentes superficiales [14].

Revista Ingenierías Universidad de Medellín 

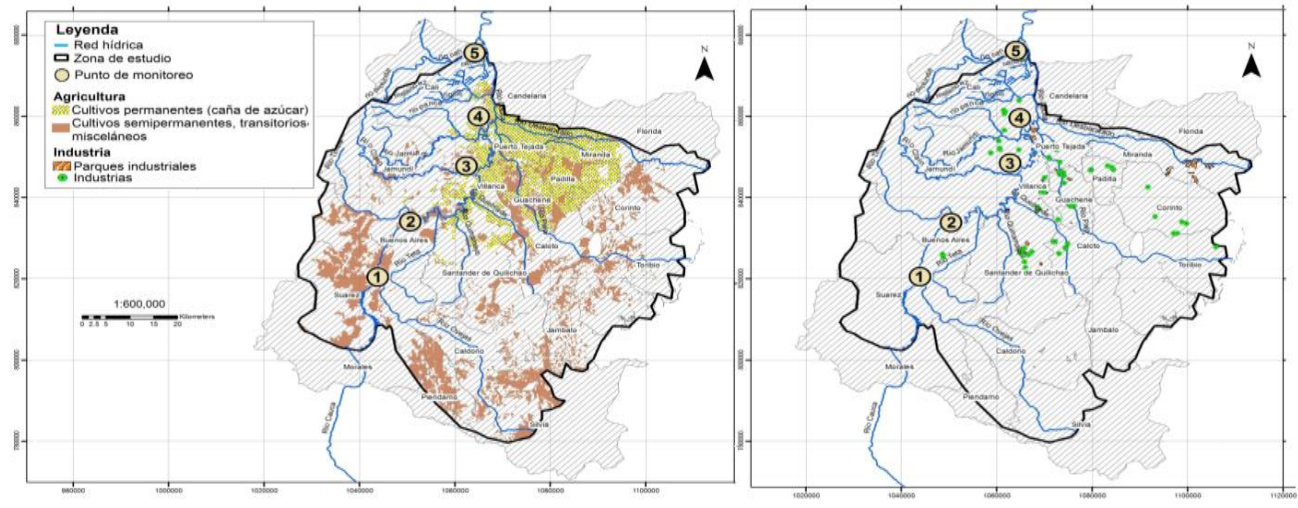

Figura 2. Mapas de uso agrícola y georreferenciación de industrias. Tramo Salvajina - Bocatoma Puerto Mallarino.

Fuente: elaboración propia

La tabla 3 detalla los tramos evaluados, las estaciones y las variables fisicoquímicas medidas en agua y sedimentos. Se incluyeron algunas variables microbiológicas y fisicoquímicas adicionales con el objetivo de calcular los índices de calidad (ICA) y de contaminación (ICOMO) $[15,16]$. Los resultados de la caracterización del agua y los sedimentos en términos de metales y plaguicidas fueron contrastados con la normativa nacional e internacional, y clasificados según la calidad de acuerdo con CETESB [17], identificando así los potenciales riesgos en la cuenca.

Tabla 3. Tramos seleccionados, estaciones y variables fisicoquímicas medidas.

\begin{tabular}{|c|c|c|}
\hline \multirow{2}{*}{ Estaciones } & \multicolumn{2}{|c|}{ Variables fisicoquímicas } \\
\hline & $\operatorname{Agua}^{*}$ & Sedimentos \\
\hline $\begin{array}{l}\text { Estación 1: antes Suárez } \\
\text { Estación 2: La Balsa } \\
\text { Estación 3: La Bolsa }\end{array}$ & $\begin{array}{l}\mathrm{pH}^{* *} \text {, temperatura } \\
\text { geno disuelto }(\mathrm{OD})^{* *} \text {, conductividinidad, cloruros, } \\
\text { color aparente y verdadero, } \mathrm{DBO}_{5} \text {, sólidos } \\
\text { totales }(\mathrm{ST}) \text {, turbiedad, dureza total, nitró- } \\
\text { geno }\end{array}$ & - \\
\hline $\begin{array}{l}\text { Estación 4: Hormiguero } \\
\text { Estación 5: Bocatoma } \\
\text { Puerto Mallarino }\end{array}$ & $\begin{array}{l}\text { totales y fecales, aluminio, antimonio, } \\
\text { arsénico, bario, benceno, boro, cadmio, } \\
\text { cianuro, cobre, cromo, hierro, manganeso, } \\
\text { mercurio, molibdeno, níquel, plata, plomo, } \\
\text { selenio, tolueno, zinc, fenoles y plaguici- } \\
\text { das }^{* * * *}\left(\text { atrazina, clorpirifos, dimetoato) }^{1} \text {, }\right. \\
\text { endosulfan }^{2}, \text { metomil }^{3} \text { y carbofuran }\end{array}$ & 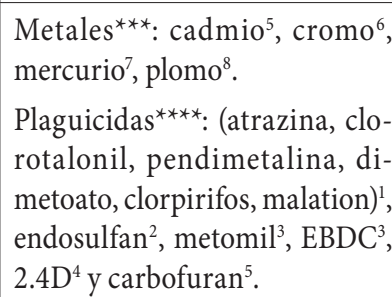 \\
\hline
\end{tabular}

${ }^{\star}[12]^{* \star}$ Mediciones in situ. ${ }^{* * *}$ Variables medidas en las estaciones 4 y 5. ${ }^{* * *}$ Plaguicidas medidos en la Estación 5. Métodos: ${ }^{1}$ EPA 8141B y 3510C. ${ }^{2}$ EPA 3550C y 3620C. ${ }^{3}$ EPA $632 .{ }^{4}$ EPA 8318. ${ }^{5}$

EPA 7130. ${ }^{6} \mathrm{EPA} 7190 .{ }^{7} \mathrm{EPA} 7471 .{ }^{8} \mathrm{EPA} 3050$.

Fuente: elaboración propia 


\section{RESULTADOS Y DISCUSIÓN}

\subsection{Construcción de la matriz de priorización de peligros químicos}

La tabla 4 muestra la matriz de priorización basada en el número de UP que utiliza el peligro químico por cada una de las 13 categorías asociadas a los procesos productivos, contrastándose con información bibliográfica que ha reportado resultados de calidad de agua en la zona de estudio.

Tabla 4. Matriz de priorización de peligros químicos asociados a los sectores productivos. Tramo de estudio Salvajina-Bocatoma Puerto Mallarino.

\begin{tabular}{|c|c|c|c|c|c|c|c|c|c|c|c|c|c|c|c|}
\hline \multirow{2}{*}{ Peligro químico } & \multicolumn{13}{|c|}{ SECTOR PRODUCTIVO* } & \multirow{2}{*}{$\begin{array}{c}\text { Total } \\
\text { UP }\end{array}$} & \multirow{2}{*}{$\begin{array}{l}\text { Información reportada } \\
\text { de calidad de agua* }\end{array}$} \\
\hline & 1 & 2 & 3 & 4 & 5 & 6 & 7 & 8 & 9 & 10 & 11 & 12 & 13 & & \\
\hline Cromo & 235 & 58 & - & 43 & 39 & - & - & 24 & - & 21 & - & - & 11 & 431 & $\begin{array}{l}\text { Excede límite reglamentario en estacio- } \\
\text { nes la Balsa, Hormiguero y Bocatoma }\end{array}$ \\
\hline Bario & 235 & 58 & 47 & - & 39 & - & - & - & - & 21 & - & - & 11 & 411 & No ha sido medido \\
\hline Arsénico & 235 & 58 & - & - & 39 & - & - & 24 & - & 21 & - & - & 11 & 388 & $\begin{array}{l}\text { No ha excedido ellímite reglamentario en } \\
\text { la Balsa, Hormiguero y bocatoma }\end{array}$ \\
\hline Benceno & 235 & - & - & - & - & - & - & - & 22 & - & 18 & - & - & 275 & No ha sido medido \\
\hline Tolueno & 235 & - & - & - & - & - & - & - & 22 & - & 18 & - & - & 275 & No ha sido medido \\
\hline 1,2dicloroetano & 235 & - & - & - & - & - & - & - & - & - & 18 & - & - & 253 & No ha sido medido \\
\hline Plomo & - & 58 & 47 & - & 39 & - & - & - & 22 & - & - & - & 11 & 177 & Excede límite reglamentario Hormiguero \\
\hline Antimonio & - & 58 & - & - & 39 & - & - & 24 & - & 21 & - & 14 & 11 & 167 & No ha sido medido \\
\hline Aluminio & - & 58 & - & - & 39 & - & 34 & - & - & 21 & - & - & 11 & 163 & $\begin{array}{l}\text { Excede límite reglamentario La Balsa } \\
\text { y Bocatoma }\end{array}$ \\
\hline Mercurio & - & 58 & - & - & 39 & - & - & - & - & 21 & 18 & - & 11 & 147 & $\begin{array}{l}\text { Excede límite reglamentario Hormigue- } \\
\text { ro, Bocatoma }\end{array}$ \\
\hline Manganeso & - & 58 & 47 & - & 39 & - & - & - & - & - & - & - & - & 144 & $\begin{array}{l}\text { Excede límite reglamentario Bocatomay } \\
\text { en todas las estaciones }\end{array}$ \\
\hline Cadmio & - & 58 & - & - & 39 & - & - & - & - & 21 & - & - & 11 & 129 & $\begin{array}{l}\text { Se mide en históricos pero el límite de } \\
\text { detección es alto referente a la norma }\end{array}$ \\
\hline Níquel & - & 58 & - & - & 39 & - & - & - & 22 & - & - & - & - & 119 & $\begin{array}{l}\text { No ha excedido el límite reglamentario } \\
\text { en las estaciones monitoreadas }\end{array}$ \\
\hline Cobre & - & 58 & - & - & 39 & - & - & - & - & - & - & - & 11 & 108 & $\begin{array}{l}\text { No ha excedido el límite reglamentario } \\
\text { Hormiguero, Bocatoma }\end{array}$ \\
\hline Selenio & - & 58 & - & - & 39 & - & - & - & - & - & - & - & 11 & 108 & $\begin{array}{l}\text { No ha excedido el límite reglamentario la } \\
\text { Balsa, Hormiguero, Bocatoma }\end{array}$ \\
\hline Hierro & - & - & 47 & - & 39 & - & - & - & - & 21 & - & - & - & 107 & $\begin{array}{l}\text { Excede límite reglamentario en la Balsa, } \\
\text { la Bolsa, Hormiguero y Bocatoma }\end{array}$ \\
\hline Fenoles & - & - & - & - & - & - & 34 & & 22 & - & 18 & 14 & - & 88 & $\begin{array}{l}\text { Excede límite reglamentario Hormigue- } \\
\text { ro, Bocatoma }\end{array}$ \\
\hline Plata & - & - & - & 43 & 39 & - & - & - & - & - & - & - & - & 82 & Excede límite reglamentario Bocatoma \\
\hline Cianuro & - & - & - & - & 39 & 38 & - & - & - & - & - & - & - & 77 & $\begin{array}{l}\text { Excede límite reglamentario en Bocatoma } \\
\text { y Estación de Juanchito }\end{array}$ \\
\hline Boro & - & - & - & - & - & - & - & - & - & 21 & 18 & - & - & 39 & No ha sido medido \\
\hline
\end{tabular}

${ }^{*}$ Convenciones: 1. Pintura, Tinte, Colorante y Laca (235 UP); 2. Metalmecánica y Metalurgia (58 UP); 3. Materiales Plásticos y Sintéticos (47 UP); 4. Fotográfica (43 UP); 5. Minería de Oro (39 UP); 6. Rallandería (38 UP); 7. Cosméticos (34 UP); 8. Curtiembre (24 UP); 9. Combustible (22 UP); 10. Farmacéutica (21 UP); 11. Química (18 UP); 12 . Papelera (14 UP); 13. Galvanoplastia (11 UP). ${ }^{* *}$ Registros históricos 2000-2009 suministrados por la entidad ambiental - CVC; [8]; [9]. 
De acuerdo con la matriz, la categoría 1 (pintura, tinte, colorante y laca) predomina en la zona, y está concentrada en el municipio de Santiago de Cali y drena al canal sur. Según la tabla de priorización, en más de 100 UP localizadas en la cuenca utilizan en su orden cromo, bario, arsénico, benceno, tolueno, 1.2 - dicloroetano, plomo, antimonio, aluminio, mercurio, manganeso, cadmio, níquel, cobre, selenio y hierro. Algunos compuestos químicos como fenoles, plata, cianuro y boro; aunque se utilizan en menos de 100 UP, en algún momento han sobrepasado los límites reglamentarios del Decreto 1594 [18] para la destinación del recurso hídrico como fuente de abastecimiento humano y doméstico.

La identificación de los peligros químicos permite recomendar que para controlar el riesgo químico en la cuenca, tanto los organismos de control ambiental como los usuarios de la fuente consideren estas variables en programas de monitoreo que les permitan evaluar de manera permanente el potencial riesgo que su presencia pueda causar y tomar medidas necesarias para evitarlo, ya que químicos como bario, benceno, tolueno, 1,2-dicloroetano y antimonio no han sido medidos y se utilizan en más de 100 UP.

Estos resultados muestran que las matrices de priorización asociadas a las actividades agrícola, minera e industrial son un primer paso para evaluar el riesgo asociado a estos peligros; sin embargo, también es recomendable considerar otros factores como el tamaño de las UP, la frecuencia de uso del producto, cantidades y concentraciones usadas en los diferentes procesos productivos y la existencia de sistemas de gestión ambiental que garanticen un manejo adecuado de estas sustancias. Adicionalmente, esta matriz de priorización puede ser un insumo importante para la construcción del mapa de riesgo del río Cauca como fuente de abastecimiento del municipio de Santiago de Cali que debe ser aplicado por municipios, autoridades ambientales y entidades prestadoras que suministren o distribuyan agua para consumo humano [19].

\subsection{Muestreo y caracterización de agua y sedimentos}

La figura 3 muestra los índices de calidad y de contaminación calculados con base en los resultados de calidad de agua.

Los índices ICA (CETESB y DINIUS) muestran una reducción en la calidad del agua a medida que el río avanza en su recorrido, por lo que requiere de tratamiento en todo el tramo evaluado para su potencial uso para consumo humano; este grado de deterioro se ratifica con el incremento progresivo del ICOMO y puede asociarse entre otras causas, a la descarga de vertimientos domésticos e industriales y escorrentía agrícola y minera que transportan sustancias tóxicas y de baja biodegradabilidad [20], como ocurre en el tramo 3 del río en el cual se concentran los impactos del sector industrial ubicado en la zona norte del departamento del Cauca y las descargas del canal sur del 

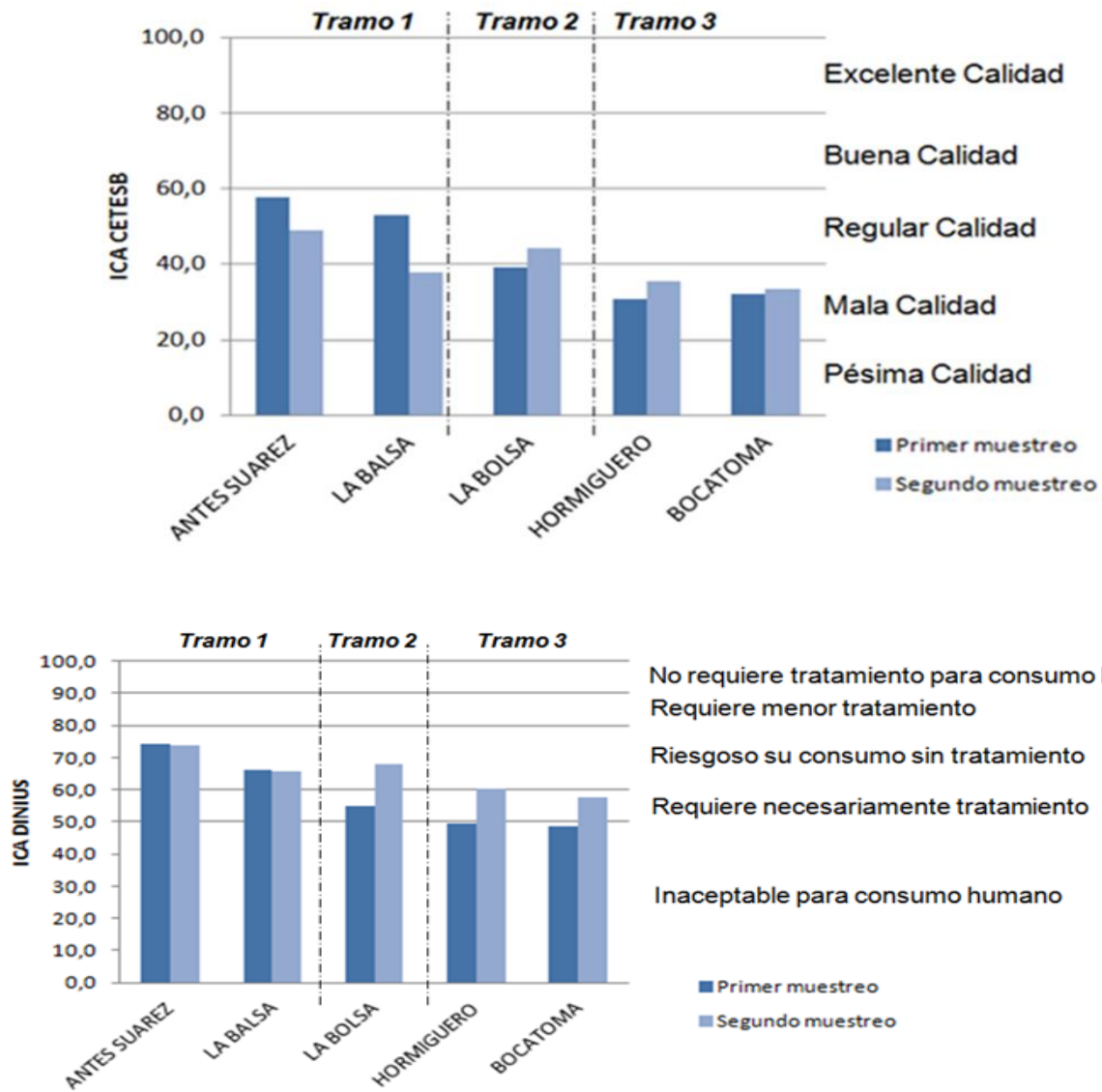

No requiere tratamiento para consumo huma Requiere menor tratamiento

Riesgoso su consumo sin tratamiento

Requiere necesariamente tratamiento

Inaceptable para consumo humano

- Primer muestreo

insegundo muestreo

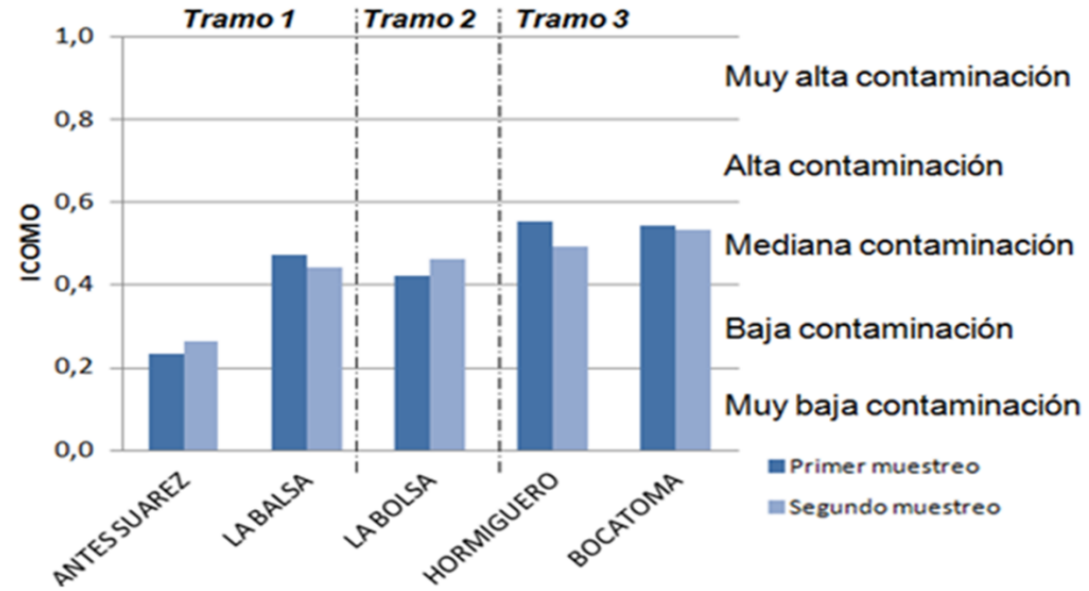

Figura 3. Índices de calidad de aqua (CETESB y DINIUS) y de contaminación (ICOMO) 
municipio de Santiago de Cali. Estas condiciones de calidad pueden incrementar la complejidad y los costos de tratamiento para potabilizar el agua, lo que sugiere que se debe ejercer prioridad en realizar un control desde la cuenca para minimizar los riesgos y reducir la complejidad de los tratamientos de potabilización.

\subsection{Metales pesados en agua y sedimentos}

En general, los metales identificados en la matriz de priorización (tabla 4) caracterizados en agua presentaron valores por debajo de los límites de detección que son inferiores a los criterios de calidad admisibles establecidos en la normativa para el uso de la fuente para consumo humano previo tratamiento; sin embargo, algunos metales pesados excedieron valores de referencia como se muestra en la tabla 5.

Tabla 5. Resultados de metales pesados identificados en la matriz de priorización medidos en agua que exceden el valor de referencia.

\begin{tabular}{|c|c|c|c|c|c|c|c|}
\hline \multirow[b]{2}{*}{ Variables } & \multirow[b]{2}{*}{ Unidades } & \multirow{2}{*}{$\begin{array}{l}\text { Valor de } \\
\text { referencia }\end{array}$} & \multicolumn{5}{|c|}{ Estaciones } \\
\hline & & & $\begin{array}{l}\text { Antes } \\
\text { Suárez }\end{array}$ & La Balsa & La Bolsa & Hormiguero & $\begin{array}{c}\text { Bocatoma Puerto } \\
\text { Mallarino }\end{array}$ \\
\hline Hierro total ${ }^{*}$ & \multirow{2}{*}{$\mathrm{mg} / \mathrm{L}$} & \multirow{2}{*}{$0,30(1)$} & 0,64 & 1,21 & 5,46 & 5,78 & 1,88 \\
\hline Hierro total ${ }^{* *}$ & & & 0,54 & 0,90 & 1,72 & 0,39 & 2,11 \\
\hline Manganeso total ${ }^{*}$ & \multirow{2}{*}{$\mathrm{mg} / \mathrm{L}$} & \multirow{2}{*}{$0,10(1)$} & 0,07 & 0,05 & 0,20 & 0,39 & 0,34 \\
\hline Manganeso total ${ }^{* *}$ & & & $<0.09$ & $<0.09$ & $<0.09$ & $<0.09$ & 0,46 \\
\hline Fenoles totales ${ }^{*}$ & $\mathrm{mg} / \mathrm{L}$ & $0,02(2)$ & 0,21 & 0,25 & 0,23 & 0,32 & 0,30 \\
\hline Boro $^{* *}$ & $\mathrm{mg} / \mathrm{L}$ & $0,50(3)$ & 0,14 & 0,18 & 0,76 & 1,32 & 0,58 \\
\hline
\end{tabular}

* Muestreo 1; ${ }^{* *}$ Muestreo 2. Normatividad: (1) Fuente Deficiente, Colombia [21]; (2) Destinación del recurso humano y doméstico, Colombia [18]; (3) Res. 357/2005, Brasil [22].

\section{Fuente: elaboración propia}

Los resultados indican que algunos peligros químicos exceden los criterios de calidad para consumo humano previo tratamiento; además, se puede observar que el boro, el cual históricamente no ha sido medido, excedió los criterios de calidad de agua, por lo que se debería considerar la medición de peligros químicos que actualmente no se tiene en cuenta en programas de monitoreo de la fuente.

Las posibles fuentes de estos metales pesados pueden ser el sector industrial que utiliza estos compuestos, las descargas del canal sur aportados por el drenaje sur de la ciudad de Cali, los lixiviados que genera el botadero de Navarro, la actividad agrícola debido a que los suelos en la parte plana del valle del río Cauca (a partir de la estación de monitoreo La Bolsa) presentan bajos niveles de manganeso (23\%), lo que requiere el uso de fertilizantes que aporten este componente y la fuente natural de los suelos $[23,24]$. La tabla 6 presenta los resultados de metales pesados en sedimentos sombrean- 
do aquellos que exceden el valor de referencia internacional "Freshwater Sediment Screening Benchmarks" [25] y clasificando su calidad de acuerdo con CETESB [17].

Tabla 6. Resultados de metales pesados en los sedimentos para las estaciones de Hormiguero y Bocatoma clasificados según CETESB [17]

\begin{tabular}{|c|c|c|c|c|c|c|c|}
\hline \multirow{3}{*}{ Variable } & \multirow{2}{*}{$\begin{array}{c}\text { Norma } \\
\text { Sedimento } \\
{[25]}\end{array}$} & \multicolumn{2}{|c|}{$\begin{array}{c}\text { Clasificación de Sedimen- } \\
\text { tos (CETESB [17]) }\end{array}$} & \multicolumn{2}{|c|}{1 Muestreo } & \multicolumn{2}{|c|}{2 Muestreo } \\
\hline & & \multirow[t]{2}{*}{1 Muestreo } & \multirow[t]{2}{*}{2 Muestreo } & Hormiguero & $\begin{array}{c}\text { Bocatoma } \\
\text { Puerto } \\
\text { Mallarino }\end{array}$ & Hormiguero & $\begin{array}{c}\text { Bocatoma Puerto } \\
\text { Mallarino }\end{array}$ \\
\hline & $m g / K g$ & & & \multicolumn{4}{|c|}{$m g / K g$} \\
\hline Cromo & 43,4 & Mala & Buena & 102 & 114 & 43,7 & 43,4 \\
\hline Cadmio & 0,99 & Mala & Buena & $<3,85$ & $<5,12$ & $<1,38$ & $<1,38$ \\
\hline Plomo & 35,8 & \multicolumn{2}{|l|}{ Óptima } & $<16,0$ & $<21,3$ & $<8,3$ & $<8,3$ \\
\hline Mercurio & 0,18 & \multicolumn{2}{|l|}{ Óptima } & $<0,08$ & $<0,09$ & 0,05 & 0,13 \\
\hline
\end{tabular}

Fuente: elaboración propia

Los metales pesados monitoreados en sedimentos no excedieron los criterios de calidad admisibles establecidos en la normativa para el uso de la fuente para consumo humano, previo tratamiento [18]; sin embargo, los valores históricos (tabla 3) mostraron que lo exceden. El coeficiente de partición sedimento/agua reportado por EPA, la baja solubilidad que los metales presentan en el agua, la tendencia hacia los medios orgánicos [26,27] y la cantidad y concentración usadas en los diferentes procesos productivos del sector industrial en la cuenca del río Cauca, demuestran que las concentraciones de los metales pueden encontrarse en otras fases diferentes al agua, lo que hace recomendable considerar la caracterización de los sedimentos como un complemento importante a los monitoreos de calidad de agua; en este caso, se evidenció acumulación de cromo y mercurio.

Los cambios químicos que se presentan en las fuentes hídricas como los procesos de óxido-reducción y disminución de $\mathrm{pH}$ pueden favorecer el aporte de los metales pesados desde los sedimentos a la columna de agua como también a través de los sólidos suspendidos, los cuales se pueden incrementar cuando ocurren temporadas de lluvias durante períodos prolongados debido a la resuspensión de sedimentos $[5,6]$.

\subsection{Plaguicidas en agua y sedimentos}

La tabla 7 muestra los resultados medidos en agua y sedimentos para plaguicidas, comparándolos con la normativa internacional. 
Identificación de peligros químicos en cuencas de abastecimiento de agua como instrumento para la evaluación ...

Tabla 7. Metales y plaguicidas medidos en agua y sedimentos

\begin{tabular}{|c|c|c|c|c|c|c|c|c|}
\hline \multirow{4}{*}{ Variable } & \multirow{3}{*}{$\begin{array}{l}\text { Norma } \\
\text { Agua }\end{array}$} & \multicolumn{2}{|c|}{ AGUA } & \multirow{3}{*}{$\begin{array}{c}\text { Norma } \\
\text { Sedimento } \\
{[25]}\end{array}$} & \multicolumn{4}{|c|}{ SEDIMENTO } \\
\hline & & \multirow{2}{*}{$\begin{array}{c}1 \text { Muestreo } \\
\text { E2 }\end{array}$} & \multirow{2}{*}{$\frac{2 \text { Muestreo }}{\text { E2 }}$} & & \multicolumn{2}{|c|}{1 Muestreo } & \multicolumn{2}{|c|}{2 Muestreo } \\
\hline & & & & & E1 & $E 2$ & E1 & $E 2$ \\
\hline & \multicolumn{3}{|c|}{$m g / l$} & \multicolumn{5}{|c|}{$m g / K g$} \\
\hline Atrazina & $0,002(1)$ & $<0,004$ & $<0,001$ & 0,00662 & $<0,4$ & $<0,4$ & $<0,10$ & $<0,10$ \\
\hline Metomil & $0,0025-0,099(2)$ & $<0,004$ & $<0,001$ & - & $<0,006$ & $<0,006$ & $<0,0014$ & $<0,0014$ \\
\hline EndosulfánI & \multirow{2}{*}{$0,00056(1)$} & $<0,00006$ & $<0,00001$ & 0,0029 & $<0,012$ & $<0,012$ & $<0,012$ & $<0,012$ \\
\hline EndosulfánII & & $<0,00008$ & $<0,00002$ & 0,014 & $<0,012$ & $<0,012$ & $<0,015$ & $<0,015$ \\
\hline Carbofurán & $0,005(3)$ & $<0,0005$ & $<0,0010$ & 0,00344 & $<0,0005$ & $<0,0005$ & $<0,0010$ & $<0,0010$ \\
\hline Dimetoato & $0,0024(4)$ & $<0,0001$ & $<0,0001$ & - & $<0,002$ & $<0,002$ & $<0,0001$ & $<0,0001$ \\
\hline Metil Clorpirifos & \multirow{2}{*}{$<0,1(5)$} & $<0,0002$ & $<0,0001$ & 0,00519 & $<0,02$ & $<0,02$ & $<0,0001$ & $<0,0001$ \\
\hline Etil Clorpirifos & & $<0,0001$ & $<0,0001$ & & $<0,01$ & $<0,01$ & $<0,0001$ & $<0,0001$ \\
\hline
\end{tabular}

E1: Estación Hormiguero; E2: Estación Bocatoma Puerto Mallarino. *Normatividad: (1) Resolução No 357 de 17 de Março de 2005. Fuentes Superficiales Clase II. Brasil [27]; (2) Concentración para causar efectos agudos en el agua potable. Methomil: Registration Review Scoping Document for Human Health Assessment [25]; (3) Norma Técnica Ecuatoriana NTE INEN 1108/2006 para agua potable [28]; (4) Concentración más alta de Dimeotato medida por el Departamento de Regulación de Pesticidas (CDPR) en fuentes superficiales [25]; (5) Datos detectados en aguas superficiales de EEUU [1].

Fuente: elaboración propia

Los resultados de plaguicidas reportados como prioritarios según el uso del suelo en la cuenca del río Cauca, exceptuando al Endosulfán I y II, indican que las concentraciones en agua no superan los valores de referencia internacionales, mientras que en los sedimentos se presentaron límites de detección mayores al valor de referencia, particularmente en la determinación de atrazina y endosulfán I; sin embargo, el hecho de que existan concentraciones tanto en el agua como en los sedimentos indica que estos se usan en la cuenca, por lo que es necesario contar con programas de muestreo y caracterización sistemáticos, puesto que los plaguicidas pueden ingresar a las fuentes hídricas de forma puntual [29].

El análisis de los plaguicidas presenta como limitante la falta de reglamentación específica en el país referente a su uso y manejo, lo que conlleva que se pueden emplear grandes cantidades de estos que posteriormente contaminan las aguas subterráneas y superficiales por escorrentía agrícola.

En el desarrollo de este estudio se pudo observar que la elaboración de la matriz de priorización de peligros químicos y el creciente deterioro del río Cauca, evidenciado en las características de agua y sedimentos medidos muestran, entre otras causas, que 
el sector industrial del norte del departamento del Cauca y los vertimientos del drenaje sur del Municipio de Santiago de Cali se concentran principalmente en el tramo 3 del río, impactando su calidad con la presencia de metales y plaguicidas.

El deterioro de la fuente puede incrementar la complejidad y los costos de tratamiento para potabilizar el agua, lo que sugiere promover la eficiente gestión del recurso hídrico como medida de control del riesgo, partiendo del concepto de considerar la cuenca como primera y principal barrera de los SAAP.

\section{CONCLUSIONES}

El análisis de los usos del suelo en la cuenca y la construcción de matrices de priorización fueron útiles para predecir la existencia de peligros químicos, y resulta prioritario el control y seguimiento de sustancias como cromo, bario, arsénico, benceno, tolueno, 1.2 - dicloroetano, plomo y 14 plaguicidas (atrazina, paraquat, picloram, ametrina, diuron, glifosato, dimetoato, metomil, clorpirifos, carbofuran, fosfamidón, metamidofos, mancozeb y carbendazim). Estos resultados constituyen un importante insumo para las autoridades ambientales y sanitarias, las cuales deben elaborar, revisar y actualizar los mapas de riesgo de las fuentes utilizadas para consumo humano, reglamentado por la Resolución 4716 de 2010 de Colombia.

El muestreo y caracterización, tanto de la columna de agua como de los sedimentos, fueron actividades complementarias, ya que los sedimentos permiten identificar la presencia de peligros que no necesariamente podrían cuantificarse en la columna de agua, como comúnmente se realiza en los programas de monitoreo; por ello es necesario contar con una eficiente y confiable cuantificación de los peligros considerando aquellos que no se han caracterizado históricamente, lo que se podría mejorar con el trabajo interinstitucional de las entidades ambientales, de salud y prestadoras del servicio de agua, optimizando los recursos con el fin de realizar programas de muestreo y caracterización sistemáticos que brinden mayor confiabilidad de los resultados.

Para la reducción de los peligros identificados en la cuenca alta del río Cauca y considerándola como principal barrera de tratamiento de un sistema de agua potable, lineamientos como los establecidos en el documento CONPES del río Cauca y la Resolución 4716 de 2010 de Colombia, podrán aportar en la orientación de estrategias de gestión del riesgo por ser dirigidos a mitigar la contaminación de la cuenca alta del río y propender por un adecuado manejo ambiental que incluye la elaboración de los mapas de riesgo.

Se recomienda la pronta expedición del proyecto de modificación de norma nacional de parámetros y valores límite máximos permisibles en vertimientos puntuales a 
cuerpos de aguas superficiales y a sistemas de alcantarillado público, el cual considera lineamientos para metales y plaguicidas, lo que permitiría realizar una evaluación del riesgo con las condiciones propias de Colombia, regulando el uso y manejo de las actividades realizadas en las cuencas.

\section{AGRADECIMIENTOS}

Los autores agradecen el apoyo a Colciencias por los programas de fomento a la formación de investigadores (Jóvenes Investigadores y Apoyo a Doctorados Nacionales) y por el apoyo financiero en la modalidad de cofinanciación con EMCALI EICE ESP y la Universidad del Valle.

\section{REFERENCIAS}

[1] WHO - World Health Organization, Guidelines for drinking-water quality. 4th edition, Geneva, Switzerland: WHO Press, 2011, 564 pp.

[2] L. Rosén, P. Hokstad, A. Lindhe, S. Sklet and J. Rostum, Generic framework and methods for integrated risk management in water safety plans, TECHNEAU report. Deliverable number. D 4.1.3, D 4.2.1, D 4.2.2, D 4.2.3, 2007, 107 pp.

[3] S. Chang and R. Doong, "Concentration and fate of persistent organochlorine pesticides in estuarine sediments using headspace solid phase microextraction”, Chemosphere, vol. 62, pp.1869-1878, 2006.

[4] M. Pérez, G. Martínez y I. Fermín, "Biodisponibilidad de metales traza en sedimentos superficiales del ecosistema lagunar costero Bocaripo-Chacopata (Península de Araya, Estado Sucre)", Boletín del Instituto Oceanográfico de Venezuela (IOV), vol. 45, n. ${ }^{\circ}$ 2, pp. 81-91, 2006.

[5] L. García - Rico, M. S. Soto - Cruz, M. E. Jara - Marini y A. Gómez - Álvarez, "Fracciones geoquímicas de $\mathrm{Cd}, \mathrm{Cu}$ y $\mathrm{Pb}$ en sedimentos costeros superficiales de zonas ostrícolas del Estado de Sonora, México", Revista Internacional de Contaminación Ambiental, vol 20, n. ${ }^{\circ}$ 4, pp. 159-167, 2004.

[6] J. P. Rodríguez, S. Achleitner, M. Möderl, W. Rauch, C. Maksimovic, N. Mcintyre, M. A. DíazGranados and M. S. Rodríguez, "Sediment and pollutant load modelling using an integrated urban drainage modelling toolbox: An application of City Drain", Water Science and Technology, vol. 61, n. ${ }^{\circ}$, pp. 2273-2282, 2010.

[7] DNP/MAVDT/MHCP-Departamento Nacional de Planeación/Ministerio de Ambiente, Vivienda y Desarrollo Territorial/Ministerio de Hacienda y Crédito Público, Documento Conpes 3624. Programa para el saneamiento, manejo y recuperación ambiental de la cuenca alta del río Cauca. Consejo Nacional de Política Económica y Social. Bogotá, Colombia, 2009.

[8] J. C. Escobar, C. Cruz, P. Torres y A. Pérez, "Impacto en el aseguramiento de la calidad del agua potable por eventos extremos de caída de oxígeno disuelto en la fuente de abastecimiento", presentado en XXXII Congreso Interamericano de Ing. Sanitaria y Ambiental AIDIS, Punta Cana-Rpca. Dominicana, 2010. 
[9] P. Torres, C. H. Cruz, P. Patiño, J. C. Escobar y A. Pérez, "Aplicación de índices de calidad de agua - ICA orientados al uso de la fuente para consumo humano", Ingeniería e Investigación, vol. 30, n. $^{\circ} 3$, pp.86-95, 2010.

[10] ICA. "Instituto Colombiano Agropecuario. Listado de registros nacionales de plaguicidas químicos de uso agrícola”, [En línea], acceso 01 de febrero, 2012; Disponible: http://www.ica. gov.co/Areas/Agricola/Servicios/Regulacion-y-Control-de-Plaguicidas-Quimicos.aspx/, 2012.

[11] L. Corra, Anexo B: Clasificación Toxicológica de los plaguicidas. En: Herramienta de capacitación para el manejo responsable de plaguicidas y sus envases: efectos sobre la saludy prevención de la exposición. 2. a ed. Buenos Aires: Organización Panamericana de la SaludOPS, 2009, $300 \mathrm{p}$.

[12] APHA, AWWA and WEF. Standard methods for the examination of water and wastewaters, 21st Edition, Washington, D, USA: APHA, 2005.

[13] C. Ramírez, J. L. García, O. Ramírez y R. Bocanegra, “Caracterización de los materiales del lecho de un río para propósitos de modelación matemática”, presentado en Evento Agua 2003, Cartagena de Indias, 2003.

[14] M. Herranz, J. P. Bolívar Raya, E. Liger Pérez; J. Payeras Socias y J. L. Pinilla Matos, Procedimiento de toma de muestras de sedimentos para determinación de radiactividad ambiental, Madrid: Colección de Informes Técnicos 11. 2007. Serie Vigilancia Radiológica Ambiental. Procedimiento 1.10, 2007, 26 pp.

[15] N. Fernández, A. Ramírez and F. Solano, "Physicochemical water quality Indices-A comparative review", Revista Bistua, vol. 1, n. ${ }^{\circ}$ 1, pp. 19-30, 2004.

[16] CETESB. “Companhia de Tecnologia de Saneamento Ambiental. Relatório de qualidade das águas interiores no estado de São Paulo. Anexo V”, [Em línea]. Acceso 12 de Octubre, 2011; Disponible: http://www.cetesb.sp.gov.br/, 2006.

[17] CETESB - Companhia de Tecnologia de Saneamento Ambiental, Índices de qualidade das águas, critérios de avaliação da qualidade dos sedimentos e indicador de controle de fontes. Qualidade das águas interiores no estado de São Paulo. Apêndice B. Brasil: Governo do estado de São Paulo-Secretaria do Meio Ambiente, 2008.

[18] MINISTERIO DE AGRICULTURA. Decreto 1594 de 1984, que reglamenta los usos del agua y residuos líquidos, República de Colombia, 1984.

[19] MAVDT - MINISTERIO DE AMBIENTE, VIVIENDA Y DESARROLLO TERRITORIAL. Resolución 4716 de 2010, que reglamenta el parágrafo del artículo 15 del Decreto 1575 de 2007 sobre Mapas de Riesgo de Calidad de Agua, República de Colombia, 2010.

[20] M. P. Ramírez, Recopilación y análisis de los diferentes parámetros de contaminación del río Cauca. Tesis de grado (Químico), Universidad del Valle, Cali, 2001, 52 pp.

[21]-RAS-- REGLAMENTO TÉCNICO DEL SECTOR DE AGUA POTABLE Y SANEAMIENTO BÁSICO, Sección II, Título C: Sistemas de potabilización, Ministerio de Desarrollo Económico-Dirección de Agua Potable y Saneamiento Básico, República de Colombia. (2000). 
[22] -CONAMA-Conselho Nacional de Meio Ambiente, Resolução N. 357 de 17 de Março de 2005, Dispõe sobre a classificação dos corpos de água e diretrizes ambientais para o seu enquadramento, bem como estabelece as condições e padrões de lançamento de efluentes, e dá outras providências, Brasil, 2005.

[23] L. M. Baena, Informe de Evaluación de Metales Pesados en los Sedimentos del Canal Navarro (Canal Interceptor Sur), Corporación Autónoma Regional del Valle del Cauca-CVC, Dirección Técnica Ambiental, 2005, 19 p.

[24] R. Duran, "Efectos de la aplicación de elementos menores en caña de azúcar en suelos del valle del río Cauca", Revista Tecnicaña, vol. 12, n. ${ }^{\circ}$ 20, pp. 18-26, 2008.

[25] EPA. "United States Environmental Protection Agency”, [En línea], acceso 04 de febrero, 2013; Disponible: http://www.epa.gov/, 2013

[26] J. D. Allison and T. L. Allison, Partition coefficients for metals in surface water, soil, and waste, EPA/600/R-05/074 Technical Report. Washington, DC: Environmental Protection Agency, 2005, 93 pp.

[27] OECD. "Organization for Economic Cooperation and Development. The Global Portal to Information on Chemical Substances, in collaboration with the European Commission (EC), the European Chemicals Agency (ECHA), the United States, Canada, Japan, the International Council of Chemical Associations (ICCA), the Business and Industry Advisory Committee (BIAC), the World Health Organization's (WHO) International Program on Chemical Safety (IPCS), the United Nations Environment Programme (UNEP) and environmental non-governmental organizations". [En línea], acceso 18 de febrero, 2012; Disponible: http:// www.echemportal.org/echemportal/index.action;jsessionid=0850E2A68BFA904FD75244B B8E03CF00/, 2012.

[28] INSTITUTO ECUATORIANO DE NORMALIZACIÓN, Norma técnica ecuatoriana para agua potable NTE INEM 1108/2006, Agua Potable-Requisitos. Quito, Ecuador, 2006.

[29] L. A. LeBlanc and K. M. Kuivila, "Occurrence, distribution and transport of pesticides into the Salton Sea Basin, California, 2001-2002”, Hydrobiologia, vol. 201, Part 3, pp. 151-172, 2008. 